\title{
A Prospective Study to Evaluate a Diagnostic Algorithm for the Use of Fluid Lymphocyte Subset Analysis in Undiagnosed Unilateral Pleural Effusions
}

\author{
Giles Dixon ${ }^{\mathrm{a}, \mathrm{b}}$ Rahul Bhatnagar ${ }^{\mathrm{a}, \mathrm{b}}$ Natalie Zahan-Evans ${ }^{\mathrm{b}} \quad$ Amelia O. Clive $^{\mathrm{a}}$ \\ Paul F. Virgo ${ }^{c}$ Mary T. Brett ${ }^{d}$ Sophie H. Otton ${ }^{e}$ Andrew R.L. Medford ${ }^{a, b}$ \\ Nick A. Maskella, b \\ ${ }^{a}$ Academic Respiratory Unit, University of Bristol, b North Bristol Lung Centre, North Bristol NHS Trust, \\ 'Department of Immunology, North Bristol NHS Trust, d Department of Cellular Pathology, North Bristol NHS Trust, \\ and ${ }^{e}$ Department of Haematology, North Bristol NHS Trust, Bristol, UK
}

\section{Keywords \\ Pleura · Lymphocytes · Flow cytology}

\begin{abstract}
Background: Haematological malignancy is an important cause of pleural effusion. Pleural effusions secondary to haematological malignancy are usually lymphocyte predominant. However, several other conditions such as carcinoma, tuberculosis, and chronic heart failure also cause lymphocytic effusions. Lymphocyte subset (LS) analysis may be a useful test to identify haematological malignancy in patients with lymphocytic effusions. However, research into their utility in pleural effusion diagnostic algorithms has not yet been published. Objectives: We aimed to determine the clinical utility of pleural fluid LS analysis and whether it can be applied to a diagnostic algorithm to identify effusions secondary to haematological malignancy. The secondary aim was to evaluate the diagnostic value of pleural fluid differential cell count. Methods: Consecutive consenting patients presenting to our pleural service between 2008 and
\end{abstract}

2013 underwent thoracentesis and differential cell count analysis. We proposed an algorithm which selected patients with lymphocytic effusions ( $>50 \%$ ) to have further fluid sent for LS analysis. Two independent consultants agreed on the cause of the original effusion after a 12-month follow-up period. Results: A total of 60 patients had samples sent for LS analysis. LS analysis had an $80 \%$ sensitivity (8/10) and a $100 \%$ specificity for the diagnosis of haematological malignancy. The positive and negative predictive values were 100 and $96.1 \%$, respectively. Overall 344 differential cell counts were analysed; $16 \%$ of pleural effusions with a malignant aetiology were neutrophilic or eosinophilic at presentation. A higher neutrophil and eosinophil count was associated with benign diagnoses, whereas a higher lymphocyte count was associated with malignant diagnoses. Conclusions: LS analysis may identify haematological malignancy in a specific cohort of patients with undiagnosed pleural effusions. A pleural fluid differential cell count provides useful additional information to streamline patient pathway decisions.

(c) 2017 S. Karger AG, Basel

\section{KARGER}

(c) 2017 S. Karger AG, Basel

E-Mail karger@karger.com

www.karger.com/res
Prof. Nick A. Maskell, DM, FRCP, FCCP

Academic Respiratory Unit, Second Floor

Learning and Research Building, North Bristol NHS Trust

Bristol BS10 5NB (UK)

E-Mail nick.maskell@bristol.ac.uk 


\section{Introduction}

Haematological cancers are amongst the commonest causes of a malignant pleural effusion $[1,2]$. Up to $16 \%$ of patients with Hodgkin and non-Hodgkin lymphoma will have a pleural effusion during their illness, occurring as either a presenting feature or later on in the disease course $[3,4]$. The mechanisms of pleural effusion include pleural infiltration by the tumour, lymphatic obstruction, secondary heart failure, renal failure, and hypoalbuminaemia [5]. Historically, the diagnosis of pleural involvement in haematological malignancy was based on simple cytological examination of pleural fluid; however, reported diagnostic rates using this method alone can be highly variable [5]. Lymphocyte subset (LS) analysis, also referred to as flow cytometry, is amongst a number of more advanced cytological tests which can improve diagnostic yield [6]. LS has been suggested as a useful investigation in pleural effusions to identify those patients with haematological malignancy, although data remain limited and there may be significant costs associated with such tests [6]. Bangerter et al. [7] looked at both ascitic and pleural fluid and found that the combined use of standard cytology and LS analysis achieved a sensitivity and specificity of $100 \%$. Despite this, there is currently no established guidance for clinicians as to where pleural fluid LS analysis may fit into a standard diagnostic algorithm.

Traditionally, cytology reports comment upon the presence or absence of visible malignant cells. When the predominant cell type in pleural fluid is also reported, patients with haematological malignancy are typically found to have lymphocytic effusions. An accurate understanding of the cellular constituents of pleural fluid can help to improve differential diagnosis and allows targeted investigations in patients with undiagnosed pleural effusions. For example, lymphocyte-predominant effusions are usually felt to warrant more invasive investigation, such as pleural biopsy, as the differential diagnosis includes malignancy and tuberculosis (TB) [8-10]. In contrast, neutrophilic effusions are more likely to represent an acute process such as infection [11].

The primary purpose of this study was to determine the effectiveness of a standardised algorithm, which focused on the role and utility of pleural LS analysis for those patients presenting with undiagnosed pleural effusions. As a secondary aim, we looked to investigate whether a differential cell count with a percentage breakdown of cellular constituents would provide any valuable additional clinical information.

Lymphocyte Subset Analysis in

Undiagnosed Unilateral Pleural Effusions

\section{Patients and Methods}

The analysis utilised prospectively collected data from patients presenting consecutively to a well-established pleural service between 2008 and 2013. Those with an undiagnosed unilateral pleural effusion were reviewed as part of a broader, actively maintained pleural database and associated study. The project received ethical approval from the South West regional ethics committee (08/ H0102/11) and was registered with the UK Clinical Trials Register (UKCRN ID 8960). All patients provided informed written consent to take part in the study and to have their details and samples stored.

As part of their initial workup, patients had pleural fluid sent for routine analysis, including cytology. The study protocol also called for the full pleural fluid differential cell count to be reported (Fig. 1). All samples were examined by experienced cytopathologists, mainly M.T.B. In those with a previous history of haematological malignancy at presentation or a clinical picture highly suggestive of haematological malignancy (such as radiological lymphadenopathy or "B" symptoms), LS analysis of the pleural fluid was also requested at presentation.

The full details of cytology reporting and LS analysis can be found in the online supplementary material (for all online suppl. material, see www.karger.com/doi/10.1159/000481290). Effusions with $>50 \%$ neutrophils or lymphocytes were categorised as neutrophilic and lymphocytic, respectively, and those with $>10 \%$ eosinophils were categorised as eosinophilic. Pleural effusions could therefore be defined simultaneously as both eosinophilic and lymphocytic or neutrophilic.

Following initial investigations, those patients with a lymphocytic effusion on cytology, but with no firm tissue diagnosis of malignancy or clear alternative diagnosis (e.g., a transudative collection in a patient with known heart failure), had a second pleural fluid sample taken (Fig. 1). This second sample was sent to a specific, experienced immunologist for LS analysis as well as for repeat cytological examination. The final diagnosis for all pleural effusions was confirmed by two independent respiratory physicians after a minimum of 12 months of follow-up (or after death). These physicians were not blinded to the LS results. All diagnoses were classified into predefined groups to facilitate further analysis. The full diagnostic criteria can be found in the online supplementary material. In those cases where there were felt to be multiple contributing factors to a pleural effusion, the likely causes were ordered to signify the greatest contributing factor first. For the purposes of this analysis, only the primary cause was used.

Statistical analysis was carried out using Microsoft Excel (2011), SPSS Statistics (9.5.0.0), and Social Science Statistics (www.socscistatistics.com).

\section{Results}

\section{Patient Demographics}

A total of 509 patients were recruited during the study period. The commonest final diagnoses were metastatic malignancy $(n=188,36.9 \%)$, infection $(n=93,18.3 \%)$, malignant mesothelioma $(n=74,14.5 \%)$, and cardiac failure ( $n=47,9.2 \%)$; 408 effusions were exudates, 61 


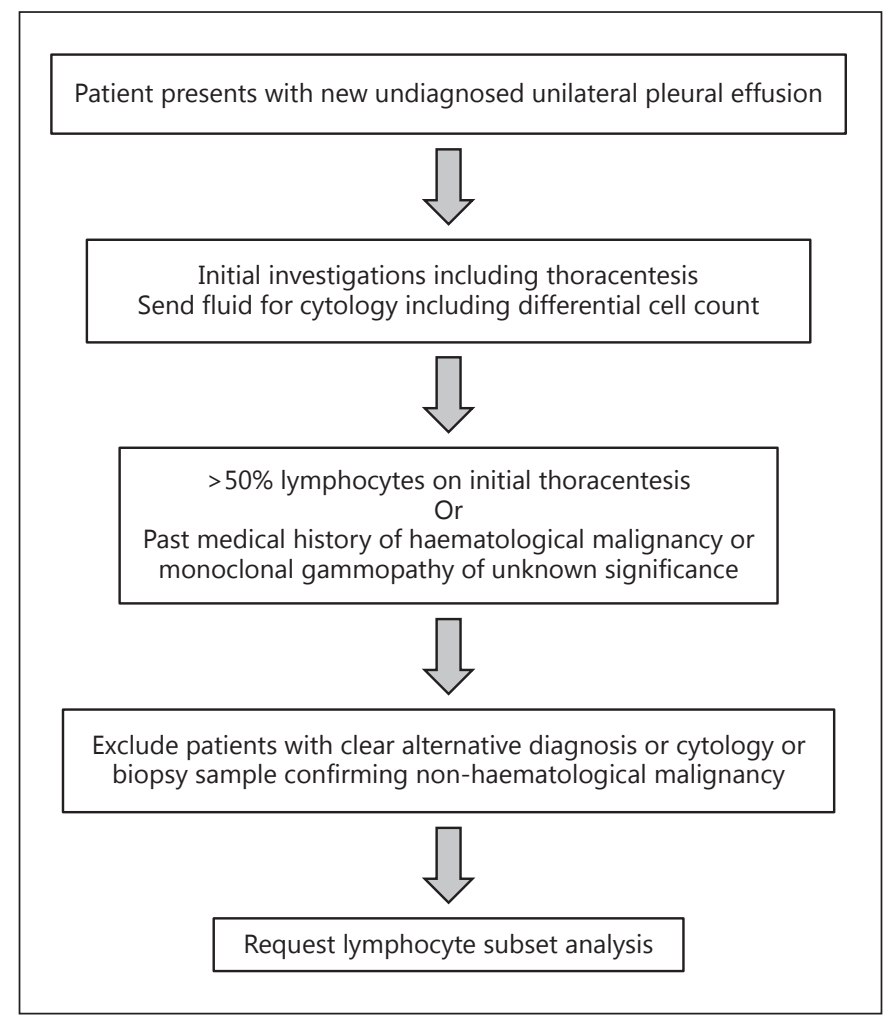

Fig. 1. Proposed algorithm for selecting patients who require lymphocyte subset analysis.

transudates, and 40 had insufficient information to enable classification. Haematological malignancy was responsible for 14 cases of pleural effusion $(2.8 \%)$ overall and $10 / 408(2.5 \%)$ exudate effusions. Other diagnoses made up the remaining 93 cases (Table 1).

\section{Clinical Utility of LS Analysis}

Pleural fluid differential cell count identified 145 patients with a lymphocytic effusion. These patients were eligible to begin the diagnostic algorithm as described above, with the outcomes demonstrated in Figure 2. During initial investigations, non-haematological malignancy was confirmed via cytology or biopsy in 56 patients, and a further 33 had a clear alternative diagnosis. One patient had a haematological malignancy confirmed at this stage by biopsy at bronchoscopy. There were therefore 63 patients who were eligible for LS analysis on repeat pleural fluid samples. Three samples were not sent for analysis or were lost in transit.

After follow-up review, the main causes for the lymphocytic effusions were metastatic malignancy $(n=58 /$ $145,40.0 \%)$, cardiac failure $(n=16 / 145,11.0 \%)$, benign
Table 1. Final diagnosis of 509 consecutive patients presenting with a unilateral pleural effusion to the pleural service at North Bristol NHS Trust between 2008 and 2013

\begin{tabular}{lc}
\hline Primary diagnosis & Frequency \\
\hline Metastatic malignancy & $188(36.9 \%)$ \\
Pleural infection & $93(18.3 \%)$ \\
Malignant mesothelioma & $74(14.5 \%)$ \\
Congestive cardiac failure & $47(9.2 \%)$ \\
Benign asbestos-related pleural effusion & $23(4.5 \%)$ \\
Inflammatory pleuritis & $20(3.9 \%)$ \\
Haematological malignancy & $14(2.8 \%)$ \\
Pleural tuberculosis & $10(2.0 \%)$ \\
Renal failure & $7(1.4 \%)$ \\
Undiagnosed at 12 months & $7(1.4 \%)$ \\
Hepatic hydrothorax & $6(1.2 \%)$ \\
Pulmonary embolism & $5(1.0 \%)$ \\
Iatrogenic/trauma & $4(0.8 \%)$ \\
Connective tissue disease & $3(0.6 \%)$ \\
Post coronary artery bypass graft & $3(0.6 \%)$ \\
Drug induced & $2(0.4 \%)$ \\
Pancreatitis & $2(0.4 \%)$ \\
Meig syndrome & $1(0.2 \%)$ \\
\hline
\end{tabular}

asbestos-related pleural effusion $(n=13 / 145,9.0 \%)$, inflammatory pleuritis $(n=12 / 145,8.3 \%)$, malignant mesothelioma $(n=12 / 145,8.3 \%)$, infection $(n=10 / 145$, $6.9 \%)$, and haematological malignancy ( $n=10 / 145$, $6.9 \%)$.

Eight out of 199 patients with non-lymphocyte-predominant effusions had LSs analysed because of a previous history of haematological malignancy or monoclonal gammopathy of unknown significance or because of clinical features strongly suggestive of haematological malignancy. One of these patients was subsequently diagnosed with an effusion secondary to diffuse large B-cell lymphoma. In this case the differential cell count showed predominantly mesothelial cells and macrophages rather than a lymphocytosis. The remaining 7 had alternative diagnoses: 3 non-haematological malignancy, 3 malignant mesothelioma, and 1 congestive heart failure.

Haematological malignancy was ultimately diagnosed in $10 / 145(6.9 \%)$ patients with a lymphocytic effusion, comprising 8 non-Hodgkin lymphomas (of which 3 were diffuse large B-cell lymphomas and 1 was a Burkitt lymphoma) and 2 chronic lymphocytic leukaemias. One out of 10 patients was diagnosed by biopsy without LS analysis (as mentioned previously).

In total, $10 / 60$ patients (16.7\%) with LS analysis performed had an effusion secondary to haematological ma- 


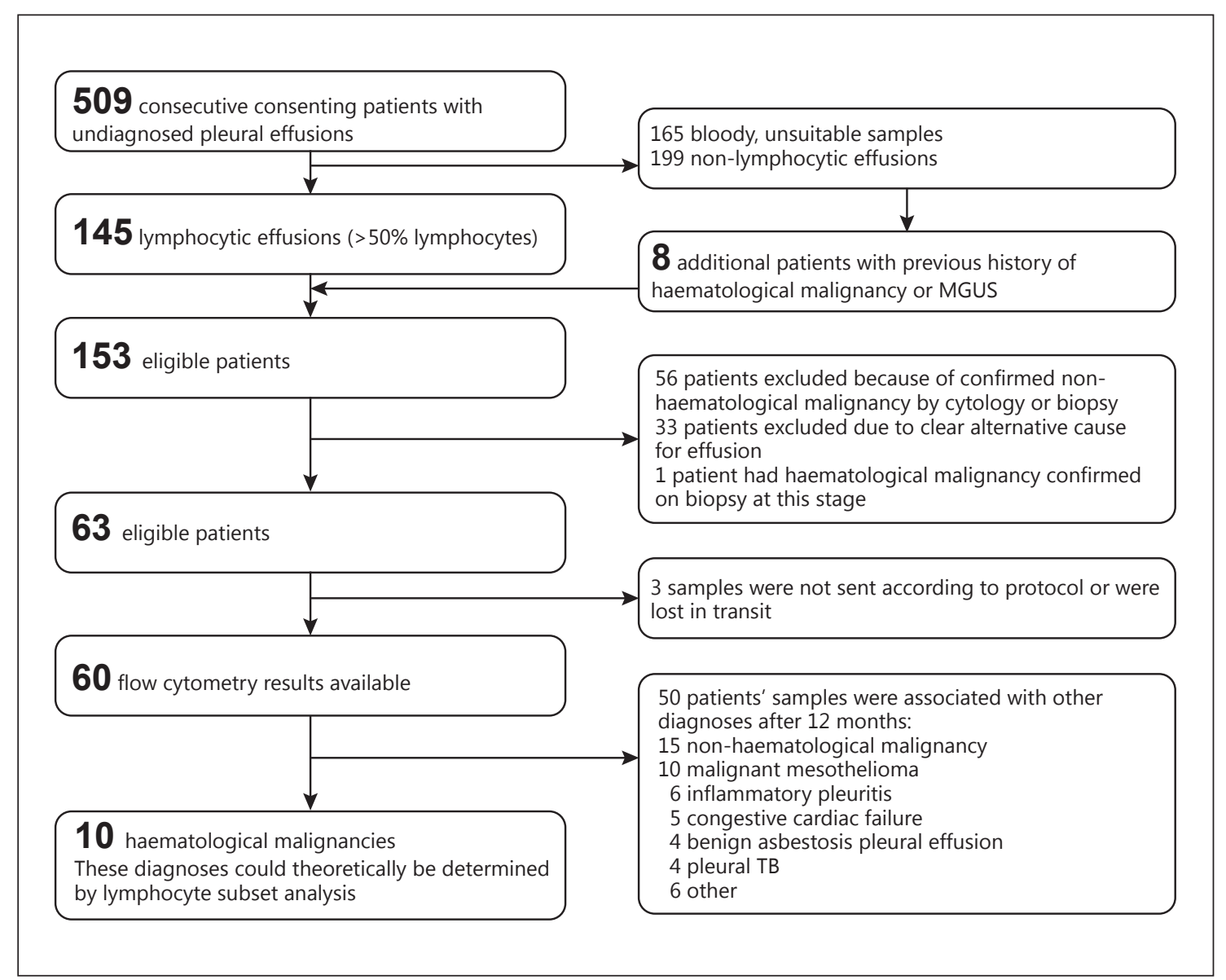

Fig. 2. Diagnostic algorithm outcomes for 509 patients presenting with a unilateral pleural effusion. MGUS, monoclonal gammopathy of unknown significance; $\mathrm{TB}$, tuberculosis.

lignancy. Four patients did not undergo LS analysis but had a subsequent diagnosis of haematological malignancy. In these cases the patients had symptoms, signs, and radiological features suggesting haematological malignancy at presentation. The patients therefore had alternative investigations to confirm their diagnosis, such as lymph node and/or bronchoscopy biopsy. The full patient details can be found in Table 2 .

LS analysis was diagnostic for haematological malignancy in 8/10 patients. Diagnostic confirmation was subsequently obtained in $9 / 10$ patients, whereby other tissue was biopsied or other fluid (ascitic/blood) sent for subset analysis.

LS analysis had a sensitivity of $80 \%$ and a specificity of $100 \%$ for the diagnosis of haematological malignancy (95\% confidence intervals $44-96$ and $91-100 \%$, respectively). The positive and negative predictive values were $100 \%$ (95\% confidence interval $60-100 \%)$ and $96.1 \%$
(95\% confidence interval 86-99\%), respectively. In the 2 patients with non-diagnostic lymphocytic effusions, the diagnosis was made by bone marrow and lymph node biopsy.

\section{A Differential Cell Count Confers Additional Useful Clinical Information}

A differential cell count was available for $344 / 509$ patients, as 165 patients had an unsatisfactory sample. Of these, as described above, there were 145 patients with $>50 \%$ lymphocytes, 54 with $>10 \%$ eosinophils, and 48 with $>50 \%$ neutrophils. Twenty-three patients had both lymphocytic and eosinophilic effusions. Therefore, 120 effusions had a mixed cellular picture which did not fit into the aforementioned categories. These included combinations of macrophages, neutrophils, lymphocytes, eosinophils, as well as malignant and mesothelial cells. 
Table 2. Characteristics of $14 / 509$ patients presenting with unilateral pleural effusion secondary to haematological malignancy

\begin{tabular}{|c|c|c|c|c|c|c|}
\hline \multirow{7}{*}{$\begin{array}{l}>50 \% \text { lymphocytes } \\
\text { on initial } \\
\text { thoracentesis }\end{array}$} & NHL (BL) & HIV & no & yes & ascitic fluid & yes \\
\hline & CLL & $\mathrm{AF}$ & yes & yes & blood & yes \\
\hline & NHL (DLBCL) & none & no & yes & lymph node & yes \\
\hline & NHL & $\mathrm{HTN}$ & yes & yes & marrow & yes \\
\hline & NHL & T2DM & yes & yes & lymph node & yes \\
\hline & NHL & IHD & yes & no & marrow & yes \\
\hline & NHL & emphysema, T2DM & yes & yes & no & no \\
\hline \multirow{3}{*}{ LS not analysed } & DLBCL & $\mathrm{T} 2 \mathrm{DM}$ & no & $\mathrm{N} / \mathrm{A}$ & bronchoscopy & yes \\
\hline & CML & none & no & N/A & $\begin{array}{l}\text { pleural fluid cytology } \\
\text { diagnostic of CML }\end{array}$ & yes \\
\hline & DLBCL & none & no & $\mathrm{N} / \mathrm{A}$ & lymph node & yes \\
\hline
\end{tabular}

AF, atrial fibrillation; BL, Burkitt lymphoma; CLL, chronic lymphocytic leukaemia; CML, chronic myeloid leukaemia; DLBCL, diffuse large B-cell lymphoma; HIV, human immunodeficiency virus; HTN, hypertension; IHD, ischaemic heart disease; LS, lymphocyte subset; NHL, non-Hodgkin lymphoma; T2DM, type 2 diabetes mellitus.

Table 3. Frequency of benign and malignant effusions of 344 patients with a unilateral pleural effusion and a percentage differential cell count

\begin{tabular}{lcc}
\hline Cell type category & Benign & Malignant \\
\hline Lymphocytic & 54 & 68 \\
Eosinophilic & 15 & 16 \\
Neutrophilic & 37 & 11 \\
Other & 40 & 80 \\
Lymphocytic and eosinophilic & 11 & 12 \\
\hline Total & 157 & 187 \\
\hline
\end{tabular}

Lymphocytic effusion defined as $>50 \%$ lymphocytes. Eosinophilic effusion defined as $>10 \%$ eosinophils. Neutrophilic effusions defined as $>50 \%$ neutrophils. "Other" includes mesothelial cells and macrophages. Lymphocytic and eosinophilic effusions contained both $>50 \%$ lymphocytes and $>10 \%$ eosinophils.

The main diagnoses of patients with lymphocytic effusions have been set out above. The main diagnoses in the 54 patients with an eosinophilic effusion were metastatic malignancy $(n=20 / 54,37.0 \%)$, malignant mesothelioma
( $n=8 / 54,14.8 \%)$, pleural infection $(n=7 / 54,13.0 \%)$, and benign asbestos-related pleural effusion $(n=6 / 54,11.1 \%)$. The main diagnoses in the 48 patients with a neutrophilic effusion were pleural infection $(n=36 / 48,75.0 \%)$, metastatic malignancy $(n=6 / 48,12.5 \%)$, and malignant mesothelioma $(n=5 / 48,10.4 \%)$.

The frequencies of benign and malignant effusions by cell type are set out in Table $3 . \chi^{2}$ comparison produced a $p$ value $<0.05$.

One hundred and eighty-seven patients with a malignant pleural effusion (including metastatic malignancy, mesothelioma, and haematological malignancy) had a differential cell count available (Fig. 3).

A benign diagnosis was found in 12/16 effusions with $>30 \%$ eosinophils compared to $15 / 25$ of effusions with values between 10 and 20\%. Highly neutrophilic effusions were more likely to be benign. However, $23 \%$ (7/30) of effusions with $>80 \%$ neutrophils were associated with malignancy. A higher lymphocyte count was associated with malignancy as effusions with $>80 \%$ lymphocytes had a $63.4 \%$ (26/41) chance of being malignant. One patient with an eosinophilic effusion had a traumatic haemothorax. 
Fig. 3. Differential cell count of 187 patients with a pleural effusion secondary to malignancy.

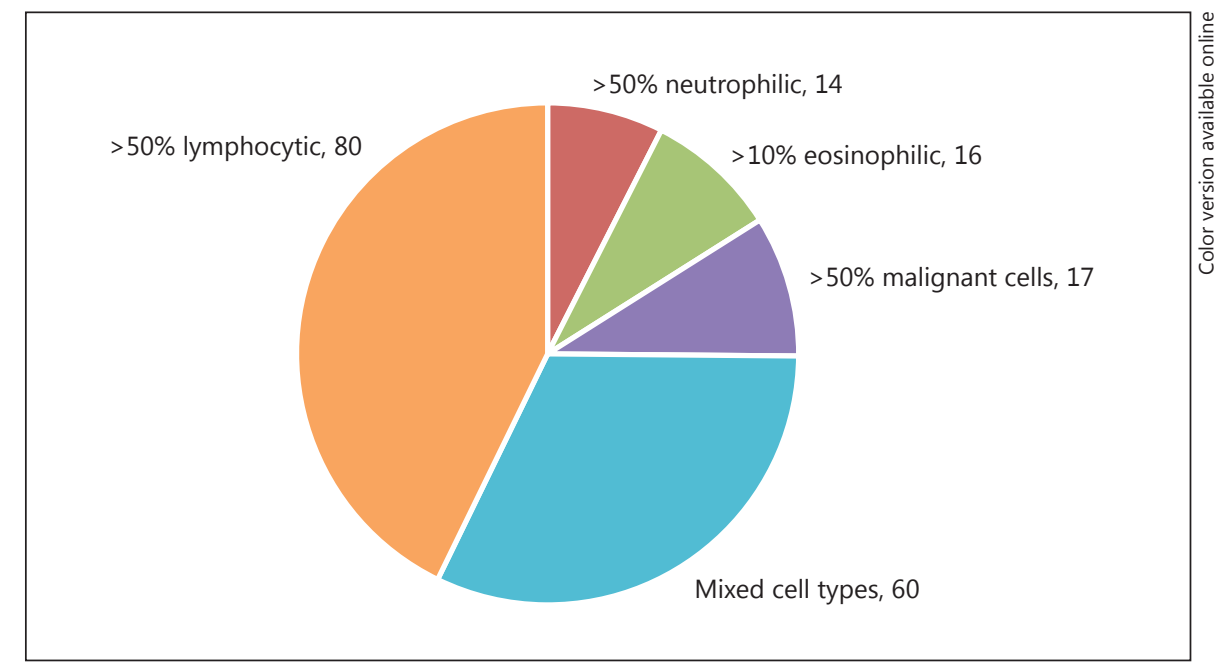

Of those patients with pleural infection and a lymphocytic effusion, $6 / 10$ patients had experienced symptoms for 2 or more weeks. Additionally, 3/10 had a concurrent diagnosis of cardiac failure, 1 patient had a concurrent diagnosis of malignancy, and 1 patient was infected with Mycobacterium avium. Of the 16 patients with $>90 \%$ lymphocytes, $75 \%(n=12)$ had effusions that were caused by malignancy.

\section{Discussion}

\section{Targeted LS Analysis}

This is the first study to prospectively analyse the use of LS exclusively in unilateral pleural effusions. In our study population, haematological malignancy was responsible for 14 pleural effusions. This relatively small incidence, coupled with the time- and labour-intensive nature of LS analysis, makes it impractical to be applied to all undiagnosed effusions. There are currently limited data exploring the clinical utility of routine LS analysis, with the most recent national guidelines unable to propose how LS analysis should be incorporated into the investigation algorithm for undiagnosed pleural effusions [6]. Those studies which have previously looked at the utility of LS analysis have not focused specifically on pleural effusions [7, 12].

Our targeted algorithm was designed to restrict LS analysis to those patients with a previous history of haematological malignancy or with undiagnosed lymphocytic effusions. This ensured that the test remained practical in day-to-day clinical use and was only applied to a group who were felt to be most likely to benefit from repeat sampling. We were able to demonstrate high sensitivity and specificity for the diagnosis of haematological malignancy, at 80 and $100 \%$, respectively. This suggests that there may be a place for the addition of LS in the routine diagnostic pathway for new pleural effusions. By following this approach, LS analysis was only required in 60/509 (11.8\%) patients presenting to our service during the study period, suggesting that it may be applied in a relatively selective manner.

In our centre differential cell count results are not usually available for $48 \mathrm{~h}$ post thoracentesis, by which time sample degradation rules out adequate LS analysis. However, if this result could be obtained more rapidly, it might allow for targeted LS analysis on the first thoracentesis sample, avoiding the need for a second procedure. We would suggest that individual centres could alter the algorithm according to their local service provision.

In patients who had LS analysis performed without a lymphocyte-predominant effusion, $1 / 8$ had haematological malignancy confirmed as the cause of their effusion. Further research is required to determine whether LS analysis is indicated within this group.

\section{Differential Cell Count Aids Diagnosis}

The standard practice in differential cell count reporting is to provide a cellular description or predominant cell type. Our work has indicated that a specific percentage differential cell count can provide useful clinical information, helping to narrow the differential diagnosis at presentation and to potentially alter management pathways. 
It has previously been reported that highly neutrophilic or eosinophilic effusions are pathognomic of benign processes $[11,13]$. However, our data suggest that an effusion with $>80 \%$ neutrophils has a one in four chance of having an underlying malignant aetiology. Whilst we found that highly eosinophilic effusions were less likely to be malignant, in 1 case we found eosinophil counts of up to $65 \%$ in a malignant effusion. Overall, we found that $16 \%$ of malignant pleural effusions in this series were neutrophilic or eosinophilic. It must therefore be stressed that highly neutrophilic or eosinophilic effusions are not always associated with a benign aetiology. One patient in this series with an eosinophilic effusion had a traumatic haemothorax, although the presence of eosinophils in pleural fluid has previously been shown to be associated with air or blood in the pleural space [14].

Lymphocytic effusions often raise diagnostic concern, especially regarding the presence of malignancy. Indeed, malignancy and TB have been reported to be the cause of around two-thirds of lymphocytic effusions in areas of moderate TB prevalence [15]. In our patient population, the prevalence of TB was much lower, with just 10/509 patients diagnosed, 6 of whom had lymphocytic effusions. Studies have previously suggested several causes of an effusion with $>80 \%$ lymphocytes, including lymphoma, rheumatoid, post-coronary artery bypass graft, malignancy, and TB [9]. Lymphocytes are also known to predominate in longstanding effusions of over 2 weeks' duration.

We have also suggested potential benefit of knowing the percentage of lymphocytes in those with lymphocytic effusions. We found that with increasing lymphocyte percentages, the likelihood of malignancy rose. Patients with highly lymphocytic effusions, in the right clinical context, should therefore be routinely considered for tissue biopsy via image-guided techniques or thoracoscopy, as well as for LS analysis.

\section{Limitations}

There are a number of limitations to our study. The primary limitation is the small number of patients with pleural effusions caused by haematological malignancy, which LS analysis is intended to detect, although the proportions found in our cohort overall are similar to those in other large-scale studies [16]. Additionally, there will always remain a group of patients in whom LS analysis may be appropriate regardless of the proposed algorithm, and these must be addressed on a case-by-case basis. Examples of these might be patients with significant undiagnosed lymphadenopathy, classical symptoms and signs of haematological malignancy, or non-specifically suspicious cytology. In our study, 4 patients were found to have haematological malignancy causing a pleural effusion and were not accommodated by the proposed algorithm.

It could also be argued that the $16.7 \%$ of patients who had the test performed and who went on to be confirmed as having a haematological malignancy is too low a proportion to justify routine clinical use. Whilst clearly not ideal, we feel that the selection of patients based upon the finding of lymphocyte-predominant fluid is the only approach which can practicably be applied to the current investigation pathways for those presenting with undiagnosed effusions.

Although our study focused on the role of lymphocytes, neutrophils, and eosinophils in pleural effusions, there are several cell types which were not present in large enough numbers frequently enough to be commented upon in this series. It has been suggested previously that effusions with $>10 \%$ basophils have an increased risk of leukaemia and are also associated with pneumothorax and pneumonia $[17,18]$. The presence of macrophages in effusions is generally thought to be non-diagnostic as they are difficult to distinguish from mesothelial cells due to overlapping morphological characteristics $[17,19]$. As mentioned above, the presence of air or blood in the pleural space has been associated with eosinophilic effusions [14]. The results presented here represent the first thoracentesis procedure carried out by the study group; however, a small number of patients may have undergone thoracentesis at a different centre prior to presentation to our pleural service, which may account for a minority of eosinophilic effusions.

A large number of patients (165/509) in our study had unsuitable pleural fluid samples and did not go on to have a differential cell count. In these patients, samples were either predominantly blood or had too few cells to enable sufficient analysis. Within the 165 patients with no differential cell count, the major diagnoses were metastatic malignancy $(n=53 / 165,32.1 \%)$, malignant mesothelio$\mathrm{ma}(n=34 / 165,20.6 \%)$, and pleural infection $(n=31 / 165$, $18.8 \%)$. The high proportion of malignancy here should reiterate the need for further investigation of this patient cohort. Despite this, 344 patients had a differential cell count reported and analysed.

Whilst the study participants were prospectively recruited to the study, the algorithm was retrospectively applied to the cohort. The participants were recruited as part of a wider study described in Patients and Methods.

Nine out of 10 patients with haematological malignancy who had LS analysis in this study also required addi- 
tional biopsy or investigation to confirm the diagnosis (Table 2). We would suggest that LS analysis diagnosed the cause of the effusion and the disease was subsequently confirmed on further investigation. Further confirmation was required to enable haematologists to make appropriate decisions regarding the treatment of the haematological malignancy.

\section{Conclusions}

LS analysis appears to have a high sensitivity and specificity for the identification of haematological malignancy as a cause of pleural effusion in those with lymphocytepredominant effusions. A differential cell count can help to guide further investigation and is helpful in the diagnosis of undiagnosed unilateral pleural effusions. Furthermore, a neutrophil- or eosinophil-predominant effusion is not always an indicator of a benign process. In addition to this, a higher percentage of pleural fluid lymphocytosis is associated with a malignant aetiology. Patients with a lymphocytic pleural effusion and no obvious alternative diagnosis after initial testing may benefit from repeat fluid sampling and LS analysis. In order to confirm the clinical utility of our proposed algorithm, further prospective analysis including a greater number of patients with effusions secondary to haematological malignancy will be required.

\section{Financial Disclosure and Conflicts of Interest}

The authors have no conflicts of interest to disclose. This research did not receive any specific grant from funding agencies in the public, commercial, or not-for-profit sectors.

\section{Author Contributions}

All authors contributed to the writing of the manuscript. M.T.B. and P.F.V. undertook cytological and LS analysis, respectively. G.D., R.B., N.Z.E., and A.O.C. were responsible for data gathering. G.D. and R.B. were responsible for data analysis. A.R.L.M. and N.A.M. were responsible for confirming each patient's final diagnosis. All authors approved this submission.

\section{References}

1 Sahn SA: State of the art. The pleura. Am Rev Respir Dis 1988;138:184-234.

2 Johnston WW: The malignant pleural effusion. A review of cytopathologic diagnoses of 584 specimens from 472 consecutive patients. Cancer 1985;56:905-909.

3 Vieta JO, Craver LF: Intrathoracic manifestations of the lymphomatoid diseases. Radiology 1941;37:138-159.

4 Das DK, Gupta SK, Ayyagari S, Bambery PK, Datta BN, Datta U: Pleural effusions in nonHodgkin's lymphoma. A cytomorphologic, cytochemical and immunologic study. Acta Cytol 1987;31:119-124.

5 Alexandrakis MG, Passam FH, Kyriakou DS, Bouros D: Pleural effusions in hematologic malignancies. Chest 2004;125:1546-1555.

6 Hooper C, Lee YC, Maskell N; BTS Pleural Guideline Group: Investigation of a unilateral pleural effusion in adults: British Thoracic Society Pleural Disease Guideline 2010. Thorax 2010;65(suppl 2):ii4-ii17.
7 Bangerter M, Hildebrand A, Griesshammer M: Combined cytomorphologic and immunophenotypic analysis in the diagnostic workup of lymphomatous effusions. Acta $\mathrm{Cy}$ tol 2001;45:307-312.

8 Sahn SA: Diagnostic value of pleural fluid analysis. Semin Respir Crit Care Med 1995; 16:269-278.

9 Yam LT: Diagnostic significance of lymphocytes in pleural effusions. Ann Intern Med 1967;66:972-982.

10 Pettersson T, Riska H: Diagnostic value of total and differential leukocyte counts in pleural effusions. Acta Med Scand 1981;210:129-135.

11 Light RW, Erozan YS, Ball WC Jr: Cells in pleural fluid. Their value in differential diagnosis. Arch Intern Med 1973;132:854-860.

12 Dunphy CH: Combined cytomorphologic and immunophenotypic approach to evaluation of effusions for lymphomatous involvement. Diagn Cytopathol 1996;15:427-430.

13 Krenke R, Nasilowski J, Korczynski P, Gorska K, Przybylowski T, Chazan R, Light RW: Incidence and aetiology of eosinophilic pleural effusion. Eur Respir J 2009;34:1111-1117.
14 Adelman M, Albelda SM, Gottlieb J, Haponik EF: Diagnostic utility of pleural fluid eosinophilia. Am J Med 1984;77:915-920.

15 Porcel JM: Pearls and myths in pleural fluid analysis. Respirology 2011;16:44-52.

16 Porcel JM, Esquerda A, Vives M, Bielsa S: Etiology of pleural effusions: analysis of more than 3,000 consecutive thoracenteses. Arch Bronconeumol 2014;50:161-165.

17 Spriggs AL, Boddington MM: The Cytology of Effusions. New York, Grune and Stratton, 1968.

18 Okimoto N, Takeyuki K, Yoshihiro H, Hideo O: Cause of basophilic pleural effusion. South Med J 2003;96:726-727.

19 Risberg B, Davidson B, Nielsen S, Dong HP, Christensen J, Johansen P, Asschenfeldt P, Berner A: Detection of monocyte/macrophage cell populations in effusions: a comparative study using flow cytometric immunophenotyping and immunocytochemistry. Diagn Cytopathol 2001;25:214-219.
Lymphocyte Subset Analysis in Undiagnosed Unilateral Pleural Effusions 